\title{
Occurrence of multi-carbapenemases producers among carbapenemase-producing Enterobacterales and in vitro activity of combinations including cefiderocol, ceftazidime-avibactam, meropenem-vaborbactam, and aztreonam in the COVID-19 era
}

\author{
Gabriele Bianco $^{1} \cdot$ Matteo Boattini ${ }^{1,2} \cdot$ Sara Comini $^{1,2} \cdot$ Roberto Casale $^{1,2} \cdot$ Marco lannaccone $^{1} \cdot$ Rossana Cavallo $^{1,2}$. \\ Cristina Costa ${ }^{1,2}$
}

Received: 7 November 2021 / Accepted: 15 January 2022 / Published online: 21 January 2022

(C) The Author(s), under exclusive licence to Springer-Verlag GmbH Germany, part of Springer Nature 2022

\begin{abstract}
Purpose To evaluate the prevalence of multi-carbapenemase-producing Enterobacterales (EB) and the activity of cefiderocol (CFDC), meropenem-vaborbactam (MEV), ceftazidime-avibactam (CZA), and combinations of CZA plus aztreonam (ATM), MEV plus ATM and CFDC plus CZA against them.

Methods A collection of carbapenemase-producing EB clinical isolates $(n=1242)$ was investigated by lateral flow immunoassay NG-Test CARBA-5 and molecular testing. Cefiderocol MICs were determined using broth microdilution Sensititre ${ }^{\mathrm{TM}}$ panel. MICs of CZA and MEV were determined by the gradient diffusion method. Antimicrobial synergy testing was performed using gradient diffusion strip crossing.

Results KPC were the most frequent carbapenemases (83.2\%), followed by VIM (9.2 \%), OXA-48-like (4.3\%) and NDM enzymes (4.1\%). Multi-carbapenemase producers were found in $10(0.8 \%)$ isolates. Three combinations of two different carbapenemases were observed: $\operatorname{KPC}+\mathrm{VIM}(n=4)$, NDM+OXA-48-like $(n=4)$, and VIM+OXA-48-like $(n=2)$. CFDC showed potent activity against eight out of ten dual-carbapenemases producers, while resistance or reduced susceptibility was shown towards CZA and MEV. CFDC in combination with CZA showed no synergistic effects and only two additive effects on seven (87.5\%) of the CFDC-susceptible strains. Conversely, CZA plus ATM and MEV plus ATM combinations were synergistic against all ATM-resistant strains regardless of dual-carbapenemases phenotype.

Conclusions The occurrence of multi-carbapenemase producers is not uncommon in Northern Italy area. MEV in combination with ATM might be considered as a potential therapeutic option, alternative to CZA plus ATM. CFDC susceptibility testing and synergy evaluation of ATM-based combinations should be performed in the lab routine to evaluate the most in vitro active antimicrobial regimen.
\end{abstract}

Keywords Carbapenemase $\cdot$ Metallo- $\beta$-lactamase $\cdot$ NG-Test CARBA-5 $\cdot$ Cefiderocol $\cdot$ Ceftazidime-avibactam · Meropenem-vaborbactam $\cdot$ COVID-19 infections

\section{Introduction}

Carbapenem-resistant Enterobacterales (CRE) rank among the top three multi-drug-resistant pathogens on WHO's priority list [1]. The subset of CRE that produces carbapen-

Gabriele Bianco

gabriele.bianco@unito.it

1 Microbiology and Virology Unit, University Hospital Città della Salute e della Scienza di Torino, Corso Bramante 88/90, 10126 Turin, Italy

2 Department of Public Health and Paediatrics, University of Torino, Turin, Italy emases, carbapenemase-producing Enterobacterales (CPE), is of high clinical and public health concern, because it has been associated with increasing mortality and high ability to spread in healthcare settings. Three major classes of carbapenemases are largely associated with the global spread of CRE: KPC (Ambler class A), metallo- $\beta$-lactamases (M $\beta \mathrm{Ls}$ ) 
(Ambler class B, e.g., NDM, VIM, and IMP), and OXA-48like (Ambler class D) carbapenemases [2].

Management and treatment of patients with infections due to CPE is a daily challenge in clinical practice since CPE strains co-harbor antimicrobial resistance determinants towards more classes of antimicrobials. Recently, several active drugs against CPE have been approved for clinical use, including $\beta$-lactam $/ \beta$-lactamase inhibitor combinations, such as ceftazidime-avibactam (CZA) and meropenemvaborbactam (MEV), and the novel siderophore cephalosporin named cefiderocol (CFDC) [3, 4].

Knowledge of carbapenemase type is important to guide antibiotic therapy since not all $\beta$-lactamase inhibitor combinations have activity against all classes of enzymes. Indeed, both avibactam and vaborbactam are potent inhibitors of class A carbapenemases but are ineffective against M $\beta$ Lsproducing strains, while only avibactam shows inhibitory activity against OXA-48-like producers [5, 6]. CFDC showed broad activity against meropenem-non-susceptible Gram-negative bacilli, including carbapenem-resistant Enterobacterales (EB), Pseudomonas aeruginosa and Acinetobacter baumannii. Its broad activity is explained by its distinctive mechanism of penetrations via the iron transport system of Gram-negative bacteria that overcomes resistance mechanisms including efflux pump upregulation and porin channel loss. Moreover, the side-chain properties render high stability against hydrolysis by $\beta$-lactamases, including serine- $\beta$-lactamases and $\mathrm{M} \beta \mathrm{Ls}$ [7].

The carriage of more than one type of carbapenemases, although not common in Europe [8], is a relevant challenge for antimicrobial treatment, especially when M $\beta$ Ls co-occur with KPC or OXA-48 like carbapenemases. Aztreonam (AZT) in combination with avibactam has shown activity against $\mathrm{M} \beta \mathrm{L}$-producing $\mathrm{EB}$, including strains co-harboring KPC or OXA-48 like carbapenemases [9-11]. However, evidence on the activity of new $\beta$-lactam/ $\beta$-lactamase inhibitor combinations and novel CFDC against multi-carbapenemases producers is limited. In this study, we evaluated the prevalence of multi-carbapenemases producers in a collection of clinical EB isolates collected from patients hospitalized during the COVID pandemic 2019-2021. Subsequently, the activity of CFDC, MEV, CZA and CZA+ATM, $\mathrm{MEV}+\mathrm{ATM}$, and CFDC+CZA combinations against multicarbapenemases producers was evaluated.

\section{Material and methods}

\section{Clinical isolates and carbapenemase detection}

CPE clinical strains were retrospectively collected from the program of surveillance and control of healthcare-associated multi-drug resistant Gram-negative infections based at our
Microbiology Laboratory. The isolates were isolated from blood, urine, rectal swabs, lower respiratory tract specimens, and wound swabs of patients admitted at the reference Centre "Città della Salute e della Scienza di Torino" and other four hospitals in Piedmont, in North-western Italy, during COVID pandemic 2019-2021. Duplicate isolates of the same species from a single patient were excluded. Species identification was carried out by matrix-assisted laser desorption ionization-time of flight mass spectrometry (MALDI-TOF MS) (Bruker DALTONIK GmbH, Bremen, Germany). Carbapenemase production was investigated by lateral flow immunoassay NG-Test CARBA 5 (NG Biotech, Guipry, France) [12]. Multi-carbapenemase producers were confirmed by Xpert Carba-R molecular assay (Cepheid, Sunnyvale, CA, USA) and were also investigated for carriage of $b l a_{\text {CTX-M-like }}$ genes (ESBL ELITe MGB Kits, ELITechGroup Molecular Diagnostics, Turin, Italy).

\section{Antimicrobial susceptibility testing}

Multi-carbapenemase-producing EB strains were subjected to antimicrobials susceptibility testing. Minimal inhibitory concentrations (MICs) of meropenem (MEM), amikacin, colistin, fosfomycin, cotrimoxazole, levofloxacin, and aztreonam were determined by a commercially available microdilution assay (Panel NMDR, Microscan WalkAway 96 Plus, Beckman Coulter, Switzerland) according to the manufacturer's instructions. Cefiderocol susceptibility was determined using Sensititre ${ }^{\mathrm{TM}}$ panel (Thermo Scientific ${ }^{\mathrm{TM}}$ ) broth microdilution panels. MICs of CZA and MEV were determined by the gradient diffusion method (Liofilchem ${ }^{\circledR}$, Roseto degli Abruzzi, Italy). All MIC values were interpreted according to the European Committee of Antimicrobial Susceptibility Testing (EUCAST) clinical Breakpoints (v.11 2021) (https://eucast.org).

\section{Evaluation of synergistic activity}

Antimicrobial synergy testing was performed on multi-carbapenemase-producing clinical strains. The following combinations of antimicrobials were evaluated for synergistic activity: CZA+ATM, MEV+ATM, CFDC+CZA.

Synergy testing was carried out using gradient diffusion strip crossing, as previously described [13]. Briefly, the strip of drug A was placed perpendicularly at a $90^{\circ}$ angle to strip off the drug B at their respective MICs onto cation-adjusted Mueller-Hinton agar plates using 0.5 MacFarland inoculum and incubated at $37{ }^{\circ} \mathrm{C}$ for $18-24 \mathrm{~h}$. MICs were read at the point at which the elliptical inhibition area touched the strips. The mean fractional inhibitory concentration index (FICI) was calculated by dividing the mean MIC of each drug in combination with the MIC of each drug alone and adding the results. The FICI results were interpreted as 
follows: $\leq 0.5$ as synergy; $>0.5$ to $\leq 4$ as no interaction; and $>4$ as antagonism.

\section{Results}

Overall, 1242 non-duplicate CPE clinical strains were collected. Of these, 1034 ( 83.2\%) produced KPC enzymes that were the most frequent carbapenemases. VIM, OXA-48like, and NDM enzymes were produced by 114 (9.2\%), 53 (4.3\%), and 51 (4.1\%) CPE strains, respectively. The most common CPE specie was Klebsiella pneumoniae (87.6\%) that was seven times more frequent than other EB species. In K. pneumoniae, the KPC enzyme (93\%) was the most prevalent carbapenemase, followed by NDM (3.4\%), VIM (2.5\%), and OXA-48-like (1.7\%). Conversely, a more homogeneous distribution of carbapenemases in the other Enterobacterales species was observed (Table 1).

Multi-carbapenemase producers were found in 10 $(0.8 \%)$ CPE isolates. Three combinations of two different carbapenemases were observed: $\operatorname{KPC}+\operatorname{VIM}(n=4)$, NDM+OXA-48-like $(n=4)$, and VIM+OXA-48-like $(n=$ 2). Concerning antimicrobial susceptibility, eight of them were resistant to both MEM and at least three other antimicrobials among amikacin, colistin, fosfomycin, cotrimoxazole, levofloxacin, and aztreonam (Table 2). The expression of a $\mathrm{M} \beta \mathrm{L}$ by all the dual-carbapenemase-producing strains also led to resistance or reduced susceptibility to the new combinations of $\beta$-lactam/ $\beta$-lactamases inhibitor. In detail, MEV showed no activity towards all strains except the KPU04 (K. pneumoniae KPC+VIM co-producer), PRW05 ( $P$. rettgeri VIM+OXA-48-like co-producer), and KPB09 (K. pneumoniae NDM+OXA-48-like co-producer), whereas CZA achieved high MICs, from 24 to $>256 \mathrm{mg} / \mathrm{L}$, for all the strains tested.

CFDC showed potent activity against all dual-carbapenemase producers except against strains KPU04 and ECR10, which tested resistant with MIC of $16 \mathrm{mg} / \mathrm{L}$.

Synergy testing results were shown in Table 3. All KPC+VIM and VIM+OXA-48-like co-producing strains tested negative for $b l a_{\mathrm{CTX}-\mathrm{M} \text {-like }}$ genes, whereas the remaining four strains were NDM+OXA-48-like and CTX-M-like co-producers. As previously observed [14], CFDC MICs determined by gradient diffusion testing were lower than those obtained with reference broth microdilution method, leading to consider strain ECR10 as false susceptible to CFDC in comparison to broth microdilution.

CFDC in combination with CZA showed no synergistic effects against dual-carbapenemase producer strains tested. However, additive effects against seven $(70 \%)$ of the tested strains were observed. Conversely, CZA+ATM and MEV+ATM combinations were synergistic against all ATM-resistant strains regardless of dual-carbapenemases phenotype. Of note, ATM reduced MIC values of both CZA and MEV below resistance breakpoint $(8 \mathrm{mg} / \mathrm{L})$ in two of ten (20\%) and seven of seven (100\%) of tested resistant strains, respectively.

Table 1 Prevalence of Enterobacterales species and carbepenemase enzymes detected during the study period (2019-2021)

\begin{tabular}{|c|c|c|c|c|c|c|c|}
\hline Prevalence & $\begin{array}{l}\text { KPC car- } \\
\text { bapenemase } \\
(\%)\end{array}$ & $\begin{array}{l}\text { OXA-48-like } \\
\text { carbapenemase } \\
(\%)\end{array}$ & $\begin{array}{l}\text { Metallo- } \beta- \\
\text { lactamase } \\
\text { VIM (\%) }\end{array}$ & $\begin{array}{l}\text { Metallo- } \beta- \\
\text { lactamase } \\
\text { NDM }(\%)\end{array}$ & $\mathrm{KPC}+\mathrm{VIM}(\%)$ & $\begin{array}{l}\text { NDM+OXA- } \\
48 \text {-like }(\%)\end{array}$ & $\begin{array}{l}\text { VIM+OXA- } \\
48 \text {-like }(\%)\end{array}$ \\
\hline $\begin{array}{l}\text { Klebsiella pneumoniae }(n= \\
1088)\end{array}$ & $1008(92.6)$ & $16(1.5)$ & $23(2.1)$ & $34(3.1)$ & $4(0.4)$ & $3(0.3)$ & \\
\hline Escherichia coli $(n=62)$ & $14(22.9)$ & $26(42.6)$ & $10(16.4)$ & $11(17.7)$ & & $1(1.6)$ & \\
\hline Enterobacter cloacae $(n=47)$ & $4(8.5)$ & & $42(89.3)$ & $1(2.1)$ & & & \\
\hline Citrobacter freundii $(n=16)$ & & & $16(100)$ & & & & \\
\hline Providencia rettgeri $(n=6)$ & & $4(66.7)$ & & & & & $2(33.3)$ \\
\hline Klebsiella oxytoca $(n=6)$ & $3(50)$ & & $3(50)$ & & & & \\
\hline Providencia stuartii $(n=5)$ & & $1(20)$ & $4(80)$ & & & & \\
\hline Klebsiella aerogenes $(n=4)$ & & & $4(100)$ & & & & \\
\hline Morganella morganii $(n=3)$ & & & $3(100)$ & & & & \\
\hline Citrobacter farmeri $(n=2)$ & & & $2(100)$ & & & & \\
\hline Proteus mirabilis $(n=1)$ & & & & $1(100)$ & & & \\
\hline Citrobacter brakii $(n=1)$ & & & $1(100)$ & & & & \\
\hline Serratia marcescens $(n=1)$ & $1(100)$ & & & & & & \\
\hline Total $(n=1242)$ & $1030(82.9)$ & $47(3.8)$ & $108(8.7)$ & $47(3.8)$ & $4(0.3)$ & $4(0.3)$ & $2(0.2)$ \\
\hline
\end{tabular}




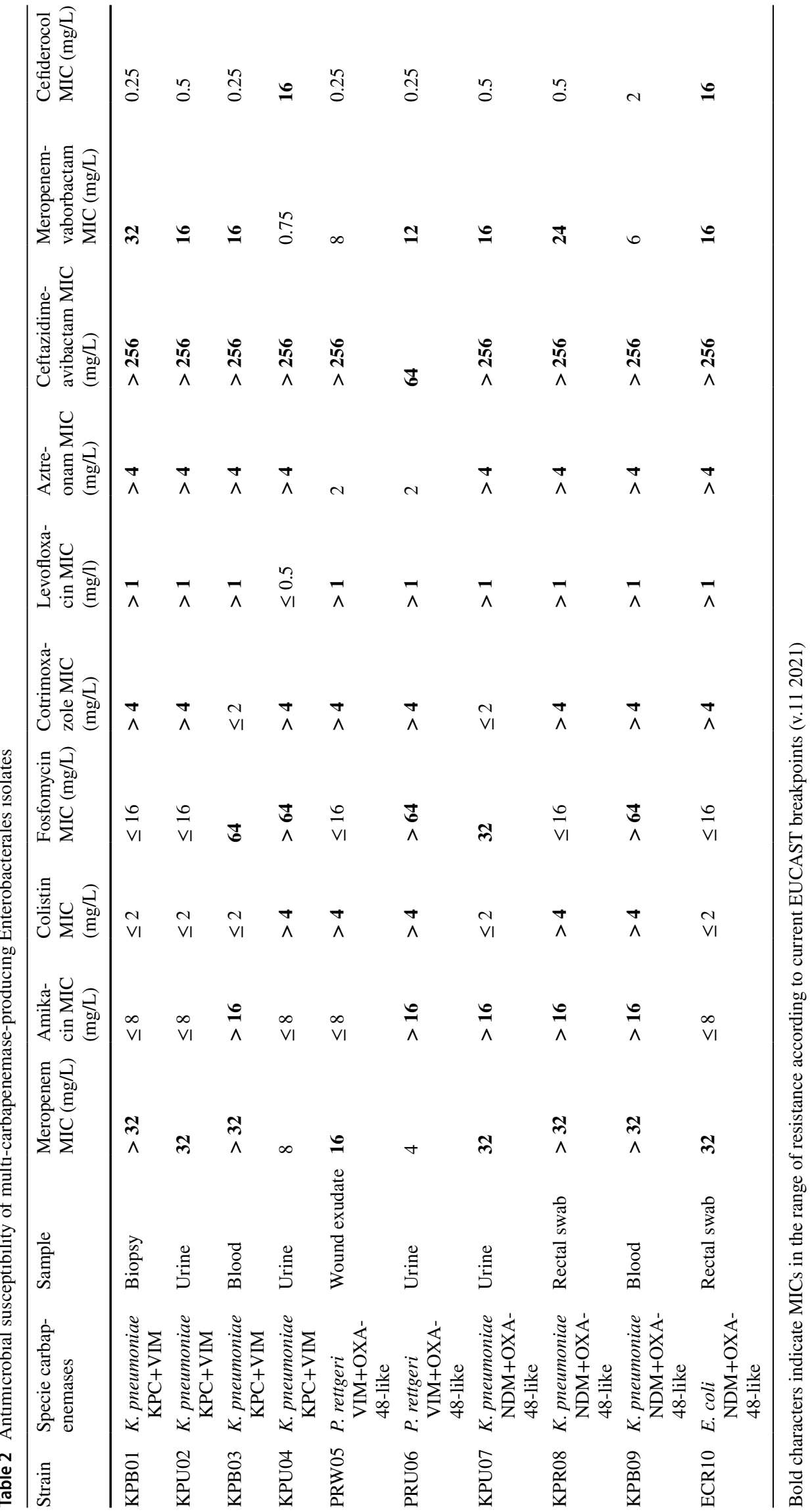




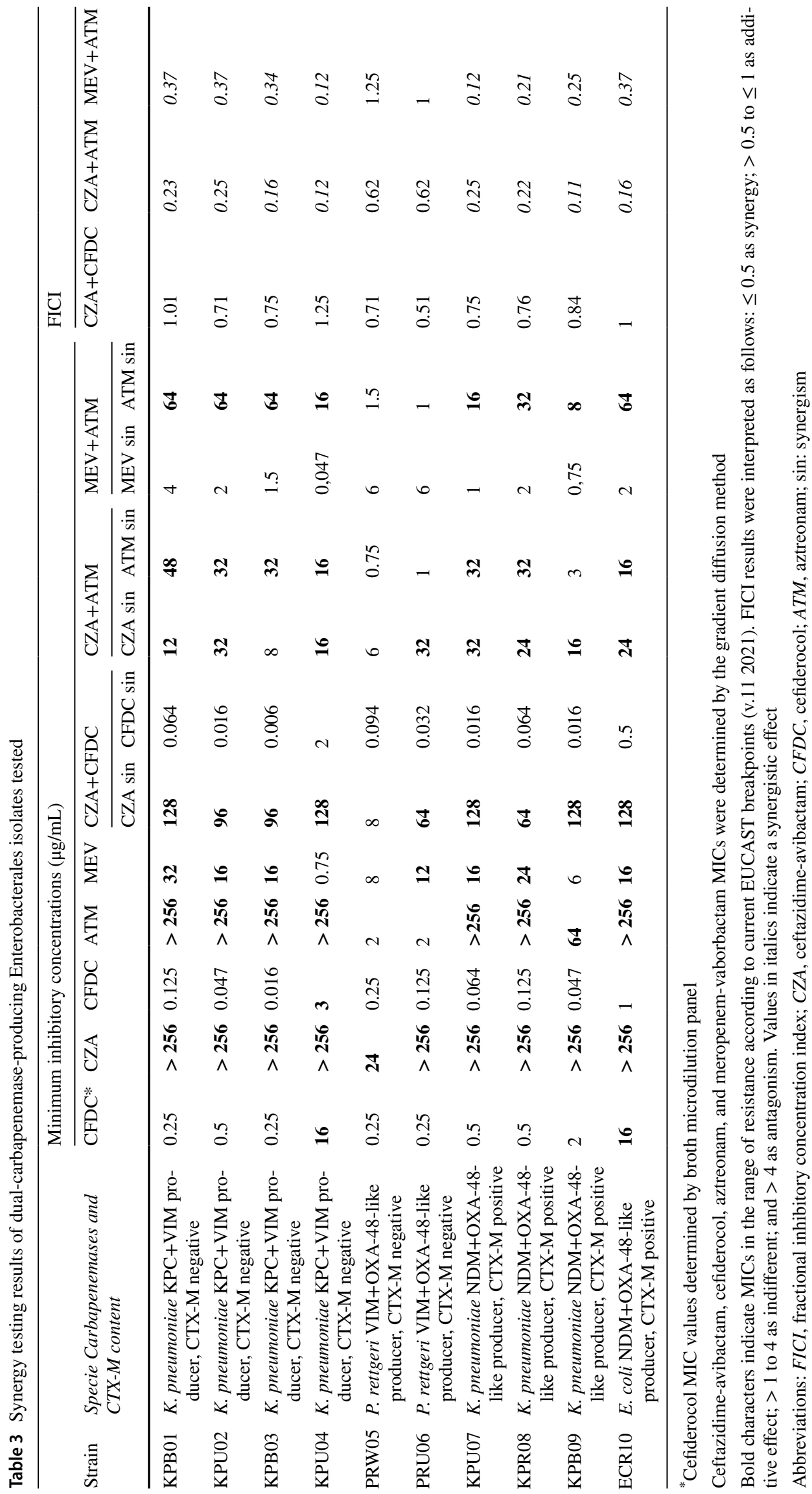




\section{Discussion}

The emergence of multi-carbapenemases producing EB has clinical, laboratory testing, and public health implications. The new combinations of $\beta$-lactam $/ \beta$-lactamase inhibitor have increased treatment options for organisms producing serine- $\beta$-lactamases (Ambler class A and D), but the growth in the proportion of isolates that co-harbor M $\beta$ Ls jeopardizes their usefulness [15]. In this study, we analyzed the prevalence of multi-carbapenemases producers in a large collection of CPE strains collected in an area of Northern Italy during a 2-year period overlapping the COVID-19 pandemic. We also evaluated in vitro activity of the new siderophore cephalosporin CFDC and the synergy of CZA+ATM, CFDC+CZA, and MEV+ATM combinations against phenotypes co-expressing $\mathrm{M} \beta \mathrm{Ls}$ and serine-carbapenemases.

The highlights of this study are the following findings: (1) prevalence of dual-carbapenemases-producing EB was notable; (2) resistance to CFDC occurred among M $\beta \mathrm{L}$ - and serine-carbapenemase-co-producing EB; (3) MEV+ATM showed synergistic activity similar to CZA+ATM against $\mathrm{M} \beta \mathrm{L}$ - and serine-carbapenemase-co-producers.

Despite KPC enzymes remain the most prevalent circulating carbapenemase type among CPE, increased frequencies of $\mathrm{M} \beta \mathrm{Ls}$ and OXA-48like enzymes in comparison to previous Italian reports have been observed $[8,16]$. In our study, a notable prevalence of multi-carbapenemases-producing EB was found, and three dualcarbapenemase-producing phenotypes were observed: KPC+VIM, NDM+OXA-48-like, and VIM+OXA-48likes. Multi-carbapenemases detection may have important microbiological and therapeutic implications. Hence, co-carriage of serine-carbapenemases and M $\beta$ Ls explained the resistance towards CZA (10/10) and MEV (7/10). Eight strains $(80 \%)$ showed resistance to ATM, related at least to the expression of serine- $\beta$-lactamases (KPC or CTX-M). CFDC showed high efficacy since eight isolates $(80 \%)$ tested susceptible with MICs ranging from 0.25 to $2 \mathrm{mg} / \mathrm{L}$. The other two dual-carbapenemase-producing strains, that were KPC+VIM-producing $K$. pneumoniae and NDM+OXA-48-like producing $E$. coli, were resistant to CFDC with MICs of $16 \mathrm{mg} / \mathrm{L}$. Although the number of strains tested was limited, CFDC plus CZA displayed no synergistic effect and additive effects on seven (87.5\%) of the CFDC-susceptible dual-carbapenemase-producing strains.

Data have emerged supporting the activity of ATM in combination with avibactam against $\mathrm{M} \beta \mathrm{L}$-producing $\mathrm{EB}$, including strains co-harboring KPC or OXA-48 like carbapenemases [9-11]. In our synergy testing evaluation, we observed that $\mathrm{CZA}+\mathrm{ATM}$ and MEV+ATM were synergistic against all ATM-resistant dual-carbapenemases-producing strains tested, both KPC+VIM and $\mathrm{NDM}+\mathrm{OXA}-48$-like+CTX-M producing phenotypes including both CFDC-resistant strains. The synergy is explained by the inhibitory activity of avibactam or vaborbactam on serine- $\beta$-lactamases (e.g., KPC and CTX-M enzymes) and refractoriness of ATM to $\mathrm{M} \beta \mathrm{L}$ hydrolysis [17]. Even if vaborbactam has no inhibitory activity on OXA-48-like enzymes, in vitro synergy between MEV and ATM against NDM+OXA-48-likes+CTX-M producers could be explained by the abovementioned mechanism together with a probably poor carbapenemase activity of OXA-48-like enzymes.

Although both CZA+ATM and MEV+ATM combinations demonstrated synergy, important considerations regarding MIC values of CZA and MEV, tested alone or combined with ATM, should be done. CZA MICs were significantly higher compared to those of MEV for all dualcarbapenemse-producing strains tested (MICs ranged from 24 to $>256 \mathrm{mg} / \mathrm{L}$ and from 0.75 to $16 \mathrm{mg} / \mathrm{L}$ for CZA and MEV, respectively). The addition of ATM highly impacted on both MEV and CZA MIC values. However, MEV MIC values decreased below the resistance breakpoint more than CZA MICs.

Our study extends existing data on the synergy between CZA and ATM, and the limited data on the synergy between MEV and ATM against M $\beta \mathrm{L}$ - and serine-carbapenemaseco-producing EB $[18,19]$. Our findings suggest that CZA and MEV may be interchangeably combined with ATM for M $\beta$ Ls and serine- $\beta$-lactamase co-producers. However, our data showed that although synergistic activities were observed for both antibiotics associations, clinically achievable concentrations were obtained more frequently for MEV in association with ATM than for CZA combined with ATM. Therefore, an association of MEV with ATM could represent an alternative to CZA plus ATM, especially in cases of selection of mutant strains resistant to avibactam. In fact, in vitro experiments showed that mutations in conserved amino acid residues of chromosomal and plasmid AmpC $\beta$-lactamase and other $\beta$-lactamases are associated with avibactam resistance in EB and Pseudomonas aeruginosa [20, 21].

Limitations of our study include the limited number of strains tested, the lack of a full characterization of $\beta$-lactamases genes content and cloning typing. Further studies including worldwide clinical isolates are necessary for the generalizability of our findings.

In conclusion, we showed that the occurrence of multicarbapenemase producers is not uncommon in Northern Italy area. Carbapenemase detection methods able to differentiate the main carbapenemase types, such as immunochromatographic or molecular assays, are recommended to identify 
multi-carbapenemase producers. Our findings suggest that MEV in combination with ATM might be considered as a potential therapeutic option, alternative to CZA plus ATM, for the treatment of infections caused by M $\beta$ Ls and serine$\beta$-lactamase co-producing EB. CFDC susceptibility testing, and synergy evaluation of ATM-based combinations should be performed in lab routine to evaluate the most appropriate antimicrobial regimen according to clinical aspects.

Data availability The authors confirm that the data supporting the findings of this study are available within the article.

Code availability Not applicable

\section{Declarations}

Ethical approval Not applicable.

Consent to participate Not applicable.

Consent for publication Not applicable.

Competing interests The authors declare no competing interests.

\section{References}

1. Tacconelli E, Carrara E, Savoldi A, Harbarth S, Mendelson M, Monnet DL, WHO Pathogens Priority List Working Group, et al. (2018) Discovery, research, and development of new antibiotics: the WHO priority list of antibiotic-resistant bacteria and tuberculosis. Lancet Infect Dis 18:318-27 https://doi.org/ 10.1016/S1473-3099(17)30753-3

2. Logan LK, Weinstein RA (2017) The epidemiology of carbapenem-resistant Enterobacteriaceae: the impact and evolution of a global menace. J Infect Dis 215:S28-S36. https://doi.org/10. 1093/infdis/jiw282

3. Wright H, Bonomo RA, Paterson DL (2017) New agents for the treatment of infections with Gram-negative bacteria: restoring the miracle or false dawn? Clin Microbiol Infect 23:704-12. https://doi.org/10.1016/j.cmi.2017.09.001

4. Tompkins K, van Duin D (2021) Treatment for carbapenemresistant Enterobacterales infections: recent advances and future directions. Eur J Clin Microbiol Infect Dis. https://doi.org/10. 1007/s10096-021-04296-1

5. Zhanel GG, Lawson CD, Adam H, Schweizer F, Zelenitsky S, Lagacé-Wiens PR et al (2013) Ceftazidime-avibactam: a novel cephalosporin/ $\beta$-lactamase inhibitor combination. Drugs 73:159-77. https://doi.org/10.1007/s40265-013-0013-7

6. Zhanel GG, Lawrence CK, Adam H, Schweizer F, Zelenitsky S, Zhanel M et al (2018) Imipenem-relebactam and meropenemvaborbactam: two novel carbapenem- $\beta$-lactamase inhibitor combinations. Drugs 78:65-98. https://doi.org/10.1007/s40265-0170851-9.Erratum.In:Drugs.2018

7. Sato T, Yamawaki K (2019) Cefiderocol: discovery, chemistry, and in vivo profiles of a novel siderophore cephalosporin. Clin Infect Dis 69:S538-S543. https://doi.org/10.1093/cid/ciz826

8. Grundmann H, Glasner C, Albiger B, Aanensen DM, Tomlinson CT, Andrasević AT et al (2017) Occurrence of carbapenemase-producing Klebsiella pneumoniae and Escherichia coli in the European survey of carbapenemase-producing Enterobacteriaceae (EuSCAPE): a prospective, multinational study. Lancet Infect Dis 17:153-163. https://doi.org/10.1016/ S1473-3099(16)30257-2

9. Sader HS, Mendes RE, Pfaller MA, Shortridge D, Flamm RK, Castanheira M (2017) Antimicrobial activities of aztreonamavibactam and comparator agents against Contemporary (2016) Clinical Enterobacteriaceae isolates. Antimicrob Agents Chemother 62:e1856-17. https://doi.org/10.1128/AAC.01856-17

10. Karlowsky JA, Kazmierczak KM, de Jonge BLM, Hackel MA, Sahm DF, Bradford PA (2017) In vitro activity of aztreonamavibactam against Enterobacteriaceae and Pseudomonas aeruginosa isolated by clinical laboratories in 40 countries from 2012 to 2015. Antimicrob Agents Chemother 61:e00472-17. https:// doi.org/10.1128/AAC.00472-17

11. Chew KL, Tay MKL, Cheng B, Lin RTP, Octavia S, Teo JWP (2018) Aztreonam-avibactam combination restores susceptibility of aztreonam in dual-carbapenemase-producing Enterobacteriaceae. Antimicrob Agents Chemother 62:e00414-18. https:// doi.org/10.1128/AAC.00414-18

12. Bianco G, Boattini M, van Asten SAV, Iannaccone M, Zanotto E, Zaccaria T, et al. (2020) RESIST-5 O.O.K.N.V. and NG-Test Carba 5 assays for the rapid detection of carbapenemase-producing Enterobacterales from positive blood cultures: a comparative study. J Hosp Infect 105:162-166 https://doi.org/10. 1016/j.jhin.2020.03.022

13. Doern CD (2014) When does 2 plus 2 equal 5? A review of antimicrobial synergy testing. J Clin Microbiol 52:4124-8. https:// doi.org/10.1128/JCM.01121-14

14. Bianco G, Boattini M, Comini S, Iannaccone M, Bondi A, Cavallo $R$ et al (2021) In vitro activity of cefiderocol against ceftazidime-avibactam susceptible and resistant KPC-producing Enterobacterales: cross-resistance and synergistic effects. Eur J Clin Microbiol Infect Dis. https://doi.org/10.1007/ s10096-021-04341-z

15. Ham DC, Mahon G, Bhaurla SK, Horwich-Scholefield S, Klein L, Dotson N et al (2021) Gram-negative bacteria harboring multiple carbapenemase genes, United States, 2012-2019. Emerg Infect Dis 27:2475-79. https://doi.org/10.3201/eid2709.210456

16. Giani T, Antonelli A, Caltagirone M, Mauri C, Nicchi J, Arena $F$ et al (2017) Evolving beta-lactamase epidemiology in Enterobacteriaceae from Italian nationwide surveillance, October 2013: KPC-carbapenemase spreading among outpatients. Euro Surveill 22:30583. https://doi.org/10.2807/1560-7917.ES.2017. 22.31.30583.Erratum.In:EuroSurveill.2017;22(34)

17. Sy SK, Beaudoin ME, Zhuang L, Löblein KI, Lux C, Kissel $M$ et al (2016) In vitro pharmacokinetics/pharmacodynamics of the combination of avibactam and aztreonam against MDR organisms. J Antimicrob Chemother 71:1866-80. https://doi. org/10.1093/jac/dkw082

18. Biagi M, Wu T, Lee M, Patel S, Butler D, Wenzler E (2019) Searching for the optimal treatment for metallo- and serine- $\beta$ lactamase producing Enterobacteriaceae: aztreonam in combination with ceftazidime-avibactam or meropenem-vaborbactam. Antimicrob Agents Chemother 63:e01426-19. https://doi.org/ 10.1128/AAC.01426-19

19. Avery LM, Mullane EM, Nicolau DP (2020) Evaluation of the in vitro activity of WCK 5222 (cefepime/zidebactam) and currently available combination therapies against single- and double-carbapenemase producing Enterobacteriaceae: expanding the zone of hope. Int J Antimicrob Agents 55:105863. https:// doi.org/10.1016/j.ijantimicag.2019.105863

20. Niu S, Wei J, Zou C, Chavda KD, Lv J, Zhang H et al (2020) In vitro selection of aztreonam/avibactam resistance in 
dual-carbapenemase-producing Klebsiella pneumoniae. J Antimicrob Chemother 75:559-65. https://doi.org/10.1093/jac/ dkz468

21. Lahiri SD, Johnstone MR, Ross PL, McLaughlin RE, Olivier NB, Alm RA (2014) Avibactam and class C $\beta$-lactamases: mechanism of inhibition, conservation of the binding pocket, and implications for resistance. Antimicrob Agents Chemother 58:5704-13. https://doi.org/10.1128/AAC.03057-14

Publisher's note Springer Nature remains neutral with regard to jurisdictional claims in published maps and institutional affiliations. 